\title{
Aus Fehlschlägen lernen - Implikationen für die Pneumologie und Intensivmedizin: Ein konzeptionelles Review
}

\author{
Learning from Failure - Implications for Respiratory and Intensive \\ Care Medicine: A Conceptual Review
}

Autor

Institut
H.-J. Kabitz

Abteilung Pneumologie (Ärztlicher Direktor: Prof. Dr. Müller-Quernheim), Universitätsklinik Freiburg eingereicht 23.5.2013 akzeptiert nach Revision 4.6.2013

Bibliografie

DOI http://dx.doi.org/

10.1055/s-0033-1344343

Online-Publikation: 11.7.2013

Pneumologie 2013; 67: 454-462

(c) Georg Thieme Verlag KG

Stuttgart · New York

ISSN 0934-8387

Korrespondenzadresse

PD Dr. Hans-Joachim Kabitz

Abteilung Pneumologie

Universitätsklinik Freiburg

Killianstraße 5

79106 Freiburg

hans-joachim.kabitz@uniklinikfreiburg.de

\section{Zusammenfassung \\ $\nabla$}

Die klinischen, gesellschaftlichen und ökonomischen Auswirkungen von medizinischen Fehlschlägen sind immens - gerade in der Pneumologie und Intensivmedizin durch besonders gravierende Ereignisse bei respiratorischer Insuffizienz, Beatmungstherapie oder Medikamentengaben. Trotz der offensichtlichen Notwendigkeit, aus (Beinahe-)Fehlschlägen zu lernen, bleibt die Umsetzung eine Rarität. Ziel dieses konzeptionellen Reviews ist es daher, Grundlagen der Terminologie, relevante Hinderungsgründe sowie mögliche Strategien aufzuzeigen, um systematisch und effektiv aus Fehlschlägen zu lernen. Basierend auf einer elektronischen Literaturrecherche (Stand: Juni 2013) unter Einbeziehung von Medline via PubMed, EMBASE, ERIC sowie Google Scholar wurden als wesentliche Hindernisgründe identifiziert: persönliche Schuldzuweisungen und das konsekutive Verheimlichen von (Beinahe-)Fehlschlägen, fehlende Analysen auf Systemebene (vs. Individualversagen) sowie (ökonomische) Fehlanreize. An wichtigen Strategien wurden identifiziert: Bedeutung des Führungsstils mit Schaffung sicherer Rahmenbedingungen, offene Berichterstattung, tragfähige Feedbackkultur, Detektionsmechanismen (z. B. „Trigger“-Werkzeuge) sowie Analyse- und Diskussionsverfahren (z.B. Zweischleifen-Lernen). Die Gründe für das Auftreten von (Beinahe-)Fehlschlägen betreffen alle Berufsgruppen im Gesundheitswesen und liegen in menschlichen, strukturellen und organisatorischen Problemfeldern. Analysen und Lösungsansätze sollten stets all diese Aspekte berücksichtigen und neben dem Individuum v. a. auch die Ebene des Systems betrachten.

\section{Abstract \\ $\nabla$}

The clinical, social and economical impact of failure in medicine [i.e., adverse health care events (AHCE)] is overwhelming. Respiratory and intensive care medicine are strongly relevant to AHCE, particularly in cases associated with respiratory failure, mechanical ventilation and pharmacotherapy. In spite of the obvious necessity to learn from AHCE, its realisation in health-care organisations is still rare. This conceptual review therefore aims to (i) clarify the most relevant terminology, (ii) identify obstacles related to this healthcare topic, and (iii) present possible strategies for solving the problems, thereby enabling respiratory and intensive care medicine to systematically and effectively learn from failure. A review of the literature (effective as of June 2013) derived from the electronic databases Medline via PubMed, EMBASE, ERIC and Google Scholar identified the following relevant obstacles (ii): a so-called blame culture associated with concealing failure, missing system analyses (vs. individual breakdown), and (economically) misdirected incentives. Possible strategies to overcome these obstacles (iii) include acknowledging the importance of leadership, a safe environment, open reporting, an effective feedback culture, and detection (e.g., trigger-tools), analysis and discussion (e.g., double loop learning) of failure. The underlying reasons for the occurrence of AHCE are based on structural, organisational and human shortcomings, and affect all categories of caregivers. Approaches to solving the problem should therefore focus primarily on the entire system, rather than on the individual alone. 


\section{Hintergrund}

\section{$\nabla$}

„Errare humanum est“ versus „Primum nihil nocere“ - auch zwei Jahrzehnte nach dem Report des Institute of Medicine aus dem Jahre 1999 [1] stehen Angehörige der Gesundheitsberufe wie auch die Politik und die breite Öffentlichkeit vor der großen Frage, wie aus Fehlschlägen im Gesundheitswesen gelernt werden kann.

Gesundheitsstörungen, welche durch die Einwirkung des Gesundheitswesens auf den Menschen/Patienten entstehen, sind von enormer klinischer, gesellschaftlicher, ökonomischer aber auch psychologischer Relevanz [2]. So zeigen Zahlen aus den USA, dass je nach Hochrechnung diese Ereignisse pro Jahr für Kosten von rund 7 Milliarden Euro sowie für rund 32000 - 98000 Todesfälle verantwortlich zu machen sind $[1,3,4]$. Komplikationen im Bereich der Beatmungs- und Intensivmedizin scheinen besonderes relevant, da sie in ihrer Ausprägung oft gravierend sind, den Krankenhausaufenthalt verlängern sowie eine erhöhte Mortalität bedingen (z.B. postoperative respiratorische Insuffizienz: +9 Tage/+22\%) [3,5-7]. Medizinische „Unerwünschte Ereignisse“ (UE; engl. adverse events) bedingen in bis $\mathrm{zu} 37 \%$ der Fälle eine intensivmedizinische Behandlung und werden bis zu 77\% als „vermeidbar“ eingestuft [4]. Während bei allen stationär behandelten Patienten die Wahrscheinlichkeit des Auftretens eines UE beachtliche 3-16\% [8-11] beträgt, erleiden 31\% der Patienten auf einer Intensivstation mindestens ein UE, welches in bis zu 29\% als „schwerwiegend“ eingestuft wird und besonders häufig das respiratorische System bzw. die Beatmungstherapie betrifft [7,12-15]. Die Wiederaufnahmerate auf eine Intensivstation beträgt bis zu $14 \%$, bedingt ein vierfach erhöhtes Mortalitätsrisiko und ist besonders häufig mit respiratorischen Komplikationen assoziiert [16,17]. Den größten unabhängigen Wiederaufnahme-Risikofaktor aller Ursachen (chirurgischer und internistischer) stellt hierbei eine vorausgegangene Beatmungstherapie während des initialen Aufenthaltes auf der Intensivstation dar [17]. Neben dem erhöhten Gefährdungspotenzial macht dies gleichzeitig deutlich, dass gerade im Bereich der (pneumologischen) Intensivmedizin die größten Chancen für relevante Verbesserungsansätze bestehen [18].

Auf einer Intensivstation treten (Beinahe-)Fehlschläge gehäuft am Vormittag (seltener gegen Mitternacht) auf, betreffen besonders häufig das respiratorische System und beruhen nicht selten auf intra- und interdisziplinären bzw. -professionellen Kommunikationsdefiziten (u.a. Schichtwechsel, getrennte Visiten etc.) $[13,19]$. Die meisten UE (ca. 20\%) sind mit Medikamentengaben assoziiert, wobei den in der Pneumologie und Intensivmedizin häufig eingesetzten Antibiotika (16\%, 1. Rang) und Antikoagulantien (11\%, 3. Rang) hierbei eine besondere Bedeutung zukommt $[18,20]$. Klinisch relevante potenzielle $(80 \%)$ und tatsächliche (20\%) medikamentöse UE auf einer Intensivstation sind häufig und betreffen die Verschreibung, die Verfügbarkeit und die Verabreichung von Medikamenten [21,22].

Häufig - besonders bei schweren UE - sind Verletzungen der Sorgfaltspflicht bzw. Nachlässigkeiten ursächlich zu beobachten [11]. Alle Berufsgruppen im Gesundheitswesen sind von Fehlschlägen betroffen; in der Intensivmedizin ist jedoch ärztliches Handeln mit einem besonders hohen Relativgewicht der beobachteten UE in Zusammenhang zu bringen $[6,18]$. Neben der Ebene des Individuums sollten Fehlschläge jedoch stets auch auf der Ebene des Systems analysiert werden [23,24].

Auch wenn die Notwendigkeit zum Lernen aus Fehlschlägen - ob klein oder groß, ob eigene oder die anderer - für das Gesund- heitswesen offensichtlich ist, sind medizinische Einrichtungen, welche diesen Anspruch effektiv und systematisch umsetzen, weiterhin eine Seltenheit [25]. Dieses konzeptionelle Review hat daher zum Ziel, Grundlagen der Terminologie, die relevanten Hinderungsgründe sowie mögliche Strategien aufzuzeigen, um systematisch und effektiv aus Fehlschlägen zu lernen.

Die Frage, wie die notwendigen Veränderungen anhand der aufgezeigten Strategien implementiert werden können, ist explizit nicht Gegenstand dieses Reviews - hier wird z.B. auf die Übersichtsarbeit von Stoller verwiesen [26].

\section{Methode \\ $\nabla$}

Die Literatursuche (Stand: Juni 2013) mit unbeschränktem Publikationszeitraum basierte auf den elektronischen (Meta-)Datenbanken Medline via PubMed, EMBASE, ERIC sowie Google Scholar $[27,28]$. Zudem wurden die Literaturverzeichnisse der Primärtreffer nach relevanten Dokumenten durchsucht. Die Suchstrategie beinhaltete die Anwendung kontrollierten Vokabulars der Datenbanken (z.B. MeSH) und zudem die Anwendung der englischen Schlüsselwörter „Learning“, „Learning from Failure“, „Medical Error“, „Critical Incident“, „Adverse (Drug) Event“, „Drug Toxicity“, „(Patient) Safety“, jeweils in Kombination mit den Begriffen „Respiratory Medicine“, „Pulmonary Medicine“ sowie „Intensive Care“. Es wurden ausschließlich Ergebnisse in deutscher und englischer Sprache berücksichtigt. Anhand der englischen Titel wurden offensichtlich nicht relevante Dokumente (z. B. Treffer mit „Failure“ i.S. eines respiratorischen Versagens) von der weiteren Analyse ausgeschlossen. Hiernach erfolgte die Selektion anhand der Zusammenfassungen der Arbeiten. Das vorliegende Review beschränkt sich auf die Abhandlung der als wesentlich erachteten Dokumente.

\section{Begriffsbestimmungen}

Exakte Begriffsbestimmungen für die Anwendung im Gesundheitswesen gestalten sich u. a. aufgrund einer treffenden Übersetzung aus dem Englischen schwierig [29], sind jedoch von entscheidender Bedeutung. Es finden sich in der Literatur diverse, zumeist geringfügige Abweichungen von den hier gewählten Definitionen für die wesentlichen Begriffe [29]. Es muss u.a. bzgl. der Definitionen beachtet werden, dass die große Mehrzahl an Fehlschlägen im Umfeld der (pneumologischen) Intensivmedizin ursächlich personenbezogen ist (63-80\%) und sich nur eine Minderheit auf technisches Versagen oder andere Ursachen zurückführen lässt $[6,15]$.

\section{Das Risiko (engl. risk)}

Ist ein Faktor (auch mehrere Faktoren), welcher die Wahrscheinlichkeit eines nicht erwünschten Endergebnis in Bezug auf die Gesundheit ansteigen lässt [30,31].

\section{Der Fehler (engl. error)}

Ist prinzipiell in seinen Ursachen vermeidbar - im Gegensatz zum Fehlschlag - und ist definiert als das Unvermögen, eine geplante (medizinische) Handlung in der Form zum Abschluss zu bringen, wie sie vorgesehen war (i.e. Fehler in der Ausführung) bzw. als die Verwendung einer falschen Strategie, um ein bestimmtes (medizinisches) Ziel zu erreichen (i.e. Fehler in der Planung) [29-31]. 
Tab. 1 Die drei verschiedenen Kategorien von Fehlschlägen und deren Kontext nach Edmondson [32].

\begin{tabular}{|ll|}
$\begin{array}{l}\text { Vermeidbare } \\
\text { Fehlschläge }\end{array}$ & $\begin{array}{l}\text { Prozessabweichungen in klar umrissenen Bereichen - } \\
\text { begünstigt durch Defizite in Verhalten, Fertigkeiten } \\
\text { oder Unterstützung } \\
\text { Kontext: } z \text {. B. Medikamentenherstellung }\end{array}$ \\
\hline $\begin{array}{l}\text { Komplexe } \\
\text { Fehlschläge }\end{array}$ & $\begin{array}{l}\text { Systemversagen aufgrund immanenter Ungewissheit - } \\
\text { können ggf. vor Eintreten eines konsekutiven Schadens } \\
\text { erkannt werden } \\
\text { Kontext: z. B. Patientenbehandlung }\end{array}$ \\
\hline $\begin{array}{l}\text { Intelligente } \\
\text { Fehlschläge }\end{array}$ & $\begin{array}{l}\text { nicht-erfolgreiche gut durchdachte Experimente - } \\
\text { enthalten zumeist wertvollen Erkenntnisgewinn } \\
\text { Kontext: z. B. Medikamentenentwicklung }\end{array}$ \\
\hline
\end{tabular}

Das Unerwünschte Ereignis (UE; engl. adverse event) Ist eine Gesundheitsstörung, welche durch eine Handlung bzw. Unterlassung des Personals im Gesundheitswesen und nicht durch eine zugrunde liegende Erkrankung des Patienten verursacht wurde; ein UE, welches einem (medizinischen) Fehler zuzuschreiben ist, wird als vermeidbares UE definiert [29-31].

Der Fehlschlag (auch: Misserfolg; engl. failure)

Stellt eine Abweichung von einem erwünschten Endergebnis dar $[25,29,32]$. Sowohl ein vermeidbares „Missgeschick“ (engl. mishap) als auch unvermeidbare Konsequenzen aus Experimenten oder anderen mit einem relevanten Risiko behafteten Interventionen werden hierunter subsumiert [25,32]. Die Anwendung dieses aufgrund der immensen Spannweite wenig präzisen Begriffes (i.e. ein Fehlschlag kann von einem winzigen Problem bis hin zu einer Katastrophe reichen) sollte behutsam und sorgfältig erfolgen, da hier immer auch eine emotionale und stigmatisierende Botschaft mitschwingt [32]. Gemäß Edmondson lassen sich die in $\bullet$ Tab. 1 dargestellten Kategorien von Fehlschlägen voneinander abgrenzen [32]. Ein Fehlschlag muss nicht zwingend zu einer Gesundheitsstörung bei einem Patienten führen im Gegensatz zum UE, welches eine solche per definitionem miteinschließt $[29,33]$. Im Folgenden werden die Begriffe UE und Fehlschlag in Abhängigkeit des Kontextes dann synonym verwendet, wenn ein Fehlschlag (mutmaßlich) in einer Gesundheitsstörung resultiert.

\section{Der Beinahe-Fehlschlag (engl. near miss)}

Ist eine Handlung bzw. Unterlassung, welche dem Patienten hätte schaden können, aber aufgrund (i) zufälliger Ereignisse bzw. (ii) präventiver Handlungen bzw. (iii) abmildernden Gegebenheiten keinen Schaden angerichtet hat $[29,34]$. Beispiele hierfür sind (i) die Applikation von Tiotropiumbromid bei bekannter AtropinÜberempfindlichkeit, ohne dass ein UE auftritt, (ii) die NichtGabe von ärztlich angeordnetem Metoprolol durch das Pflegepersonal bei bekanntem schwerem Asthma bronchiale und (iii) die Protamin-Gabe nach inzidentieller Heparin-Überdosierung.

\section{Hinderungsgründe, um im Gesundheitswesen aus Fehlschlägen zu lernen \\ $\nabla$}

In diesem Abschnitt werden relevante Hinderungsgründe abgehandelt, welche im Gesundheitswesen verhindern, dass effektiv aus Fehlschlägen gelernt werden kann.

\section{Schuldzuweisungen/Bestrafung von Fehlschlägen}

Anerkennung durch Vorgesetzte und Kollegen ist für die meisten Menschen von immenser Wichtigkeit und wird (vermeintlich!) durch persönliche (Beinahe-)Fehlschläge gefährdet; dies verleitet besonders in der Intensivmedizin [35] dazu, diese nicht zu enthüllen oder öffentlich anzuerkennen, und bedingt ein überschätztes Selbstbild („positive Illusion“) [24,32,36]. In der Folge sind persönliche Schuldzuweisungen, häufig in Form einer stereotypen Abfolge aus „personalisieren, beschuldigen, sich schämen“ (engl. „name, blame, shame“) eine weit verbreite Form des Umgangs mit Fehlschlägen [24,32,37].

Die Angst, persönlich für einen Fehlschlag verantwortlich gemacht zu werden, ist einer der wichtigsten Gründe, warum UE nicht gemeldet werden [31]. Auch die journalistische Berichterstattung über (Beinahe-)Fehlschläge im Gesundheitswesen sowie Schilderungen Betroffener verdeutlichen die tiefsitzende Kultur, Fehlschläge mit persönlichen Schuldzuweisungen zu verknüpfen $[38,39]$. Individuelle Schuldzuweisungen sind die wohl hartnäckigste und alles durchdringende Gefährdung der Sicherheitsstruktur eines Unternehmens [37]. Sie beruhen v.a. auf psychologischen Phänomenen wie dem Attributionsfehler (der Einfluss des Individuums wird gegenüber situativen Einflüssen vehement überschätzt), der Illusion des freien Willens (die menschliche Handlung wird stets als entscheidend und "gewählt" angesehen), dem Gerechte-Welt-Glauben („Jeder bekommt, was er verdient“) sowie dem Rückschaufehler (,ich hab es doch gleich gesagt") [37].

\section{Systemversagen versus Individualversagen}

Das „Schweizer-Käse-Modell“ visualisiert den Grundgedanken komplexer Institutionen wie z.B. Krankenhäusern: Reiht man mehrere Käsescheiben hintereinander, existieren zahllose versetzt liegende Löcher (i.e. Beinahe-Fehlschläge etc.) innerhalb der einzelnen Scheiben - lediglich eine sehr geringe Anzahl der Löcher lässt sich jedoch gleich einem „zufälligen“ Tunnel durch alle Schichten (i. e. eingetretenes UE) verfolgen [40].

Lediglich $2-5 \%$ der beruflichen Fehlschläge werden als „durch schuldhafte Handlungen verursacht" eingeschätzt - aber 70-90\% werden so behandelt [41]. Häufig wird außer Acht gelassen, dass in der Einschätzung von Fehlschlägen ein kontinuierlicher Übergang von tadelnswert (z.B. vorsätzliches Fehlverhalten) bis lobenswert (z.B. missglücktes wissenschaftliches Experiment) besteht [41]. Fehlschläge werden in vielen Fällen lediglich auf der Ebene des Individuums und nicht zusätzlich auf der Ebene des Systems analysiert [23]. Hier geben nach Charles Perrow die Rahmenbedingungen sowohl den Handlungsspielraum der Personen als auch die Konsequenzen von unausweichlich auftretenden (Beinahe-)Fehlschlägen vor [23, 42]. In einem solchen System hängt alles eng zusammen und es besteht eine interaktive Komplexität (i.e. zwei oder mehr (Beinahe-)Fehlschläge interagieren auf unvorhersehbare Art und Weise), welche verborgene Interaktionen bedingt (i.e. die direkten Konsequenzen des eigenen Handelns bleiben unverstanden) [23,42]. Eine krankende Infrastruktur bedingt bei dem "Syndrom des vulnerablen Systems“ mit den Komponenten 1. Schuldzuweisung, 2. Verleugnung und 3. Fehlanreize dessen Anfälligkeit gegenüber Fehlschlägen [37].

\section{Die Schwierigkeit, über Fehlschläge zu sprechen}

Persönliche Schuldzuweisungen vereiteln die Chance, Unzulänglichkeiten im System aufzudecken, und fördern Verleugnung [37]. Sogenannte „pathologische“ Institutionen machen Hinweisgeber, die auf potenzielle Gefahren oder Schwachstellen im Sys- 
tem hinweisen wollen (engl. whistle-blower), mundtot, verleumden sie, stoßen sie aus, vertuschen und/oder bestrafen Fehlschläge, ersticken neue Ideen und schaffen kein gemeinsames Sicherheitsverständnis [37]. Plakativ gesprochen: Derartige Einrichtungen wollen nicht lernen - und tun es offensichtlich auch nicht $[25,37]$. In abgeschwächter Form handeln „bürokratische“ Einrichtungen bei Fehlschlägen derart, dass sie diese als Einzelfall darstellen und Systemreformen scheuen; neue Ideen werden als Problem - statt als Chance - begriffen [37]. Gerade im Gesundheitswesen hindert das vorherrschende Klima (z.B. persönliche Schuldzuweisungen etc.) die Beteiligten häufig daran, Bedenken offen vorzutragen, um (Beinahe-)Fehlschläge aufzuspüren, bevor Patienten zu Schaden kommen [23-25].

\section{Strategien, um im Gesundheitswesen aus Fehlschlägen zu lernen}

Im folgenden Abschnitt werden nun Lösungsansätze vorgestellt, welche im Gesundheitswesen ein effektives Lernen aus Fehlschlägen ermöglichen können.

\section{Schaffung sicherer Rahmenbedingungen}

Anstelle persönlicher Schuldzuweisungen sollten die situativen und System-Einflüsse analysiert und thematisiert werden (Vermeidung des Attributionsfehlers) [43]; ein besseres Grundverständnis lässt sich v.a. durch die Erkenntnis fördern, dass auch den besten Mitarbeitern die schlimmsten Fehlschläge unterlaufen können [37]. Bei UE dürfen nicht nur die finalen Handlungen (oder Unterlassungen) am Patienten (i.e. die „Spitze des Speers“) analysiert werden, sondern es muss die gesamte Kausalkette und deren Einbettung im System (i.e. das „stumpfe Ende des Speers“) kritisch hinterfragt werden [37]. Letztlich ist es trotz der intuitiven (nicht haltbaren) Gegenargumente bedeutend einfacher und zielführender, situative Änderungen im Gesamtsystem vorzunehmen, als die Persönlichkeit der Mitarbeiter umzugestalten [37]. Die Schaffung glaubhafter, sicherer Rahmenbedingungen mit der Gewissheit, dass (Beinahe-)Fehlschläge weder institutionell noch gesellschaftlich oder juristisch bestraft werden, aber dennoch die Grenzen jedem Einzelnen klar bekannt sind (z. B. Kompetenzüberschreitungen), ist eine Grundvoraussetzung, um hieraus als Team - nur als solches - eine tragfähige, lernfreudige Umgebung zu gestalten $[23,44,45]$. Eine solche Umgebung erleichtert z. B. die Umsetzung von Richtlinien zur verbesserten Patientenversorgung (engl. best practice) auf Intensivstationen [46].

Offene Berichterstattung über „Interna“ und Bedenken Die Art und Weise, wie Einrichtungen mit sicherheitsrelevanten Informationen umgehen, determiniert deren Ausrichtung: Eine fruchtbare Atmosphäre motiviert das Personal zu beobachten, nachzufragen, eigene Schlussfolgerungen offen auszusprechen, und Vorgesetzte auf wichtige Beobachtungen hinzuweisen [37, 47]. Durch die Schaffung sicherer Rahmenbedingungen gelingt es, unter allen Beteiligten eine offene, freiwillige Berichterstattung in Bezug auf (Beinahe-)Fehlschläge zu fördern [23]. Um aus Fehlschlägen im Gesundheitswesen lernen zu können, müssen Einrichtungen dem Überbringer (vermeintlich) schlechter Nachrichten Hochachtung entgegenbringen - anstatt ihn zu bestrafen $[25,32,37]$.
Tab. 2 Rahmendbedingungen für effektives Feedback im klinischen Umfeld in Anlehnung an Ramani und Krackov [51].

Schaffen einer lernfreudigen Umgebung
Kommunizieren von Zielvorstellungen
Basieren auf direkter Beobachtung
Durchführung zeitnah \& regelmäßig
Beginnen mit der Selbsteinschätzung
Festigen/Korrigieren von Verhaltensmustern
Verwenden spezifischer \& neutraler Sprache
Bestärken des Gelernten
Abschließen mit einem Handlungsplan
Reflektieren der eigenen Fertigkeiten
Fördern der Personalentwicklung

\section{Eine tragfähige Feedbackkultur etablieren}

Oben genannte Rahmenbedingungen ermöglichen aufrichtiges, offenes Feedback zu geben und schwierige Gesprächssituationen i. S. eines produktiven Konflikts (engl. productive conflict) zu gestalten, ohne um den heißen Brei herum reden zu müssen [32, $48,49]$. In einem lernfreudigen Umfeld $[44,50]$ ist Feedback bidirektional: Die Führungskräfte geben Feedback in Form von Coaching und Empfehlungen - das Behandlungsteam gibt Feedback anhand der täglichen Erfahrungen „an vorderster Front“ [32, 48]. Essenziell ist gerade im klinischen Umfeld, dass zurechtweisendes, unidirektionales Feedback („Sie müssen das richtig machen") vermieden wird und weitere Rahmenbedingungen eingehalten werden; die wichtigsten Aspekte hierzu fasst $\bullet$ Tab. 2 zusammen $[48,51]$.

\section{Die Bedeutung des Führungsstils und}

des Behandlungsteams

Der Führungsstil bzw. die Führungskräfte (engl. leadership) sind maßgebend dafür, ob es gelingt ein Klima des offenen und produktiven Umgangs mit (Beinahe-)Fehlschlägen zu schaffen [23, 25]. Ferner muss es Führungskräften gelingen, eine überzeugende und tragfähige Vision aufzubauen, welche es den Beschäftigten ermöglicht, kreativ und gestaltend tätig zu werden [25,52]. Verblüffend schienen zunächst die Ergebnisse, dass in Pflegeteams mit einem guten Führungsstil und guter Teamarbeit mehr - anstatt weniger - (Beinahe-)Fehlschläge nachgewiesen werden konnten und dies nicht etwa auf komplexere Behandlungsfälle zurückzuführen war $[23,25]$. Die Auflösung des vermeintlichen Paradoxons besteht am ehesten darin, dass nur in einem offenen und sicheren Klima - welches maßgeblich durch den Führungsstil geprägt wird - (Beinahe-)Fehlschläge auch tatsächlich angesprochen werden [25]. Im Umkehrschluss impliziert dies, dass bei häufigen (kommunizierten!) (Beinahe-)Fehlschlägen besser funktionierende Behandlungsteams zu vermuten sind als bei seltenen [23-25]. Sichere Rahmenbedingungen und ein tragfähiger, teamorientierter Führungsstil sind auf der Intensivstation nachweislich in der Lage, die Verweildauern zu verkürzen, die Fluktuation des Pflegepersonals zu reduzieren und die Behandlungsqualität zu verbessern $[53,54]$.

\section{Möglichkeiten fördern, um Fehlschläge aufzuspüren}

Es müssen Rahmenbedingungen geschaffen werden, welche es ermöglichen, zuverlässig und zeitnah Fehlschläge jedweder Art und jeden Ausmaßes aufzuspüren. Die $\bullet$ Abb. 1 zeigt die wichtigsten in der Literatur identifizierten Schwachstellen für potenzielle UE im Zusammenhang mit Medikamentengaben [18,23]. Erst der Einsatz möglichst vieler komplementärer Verfahren um 


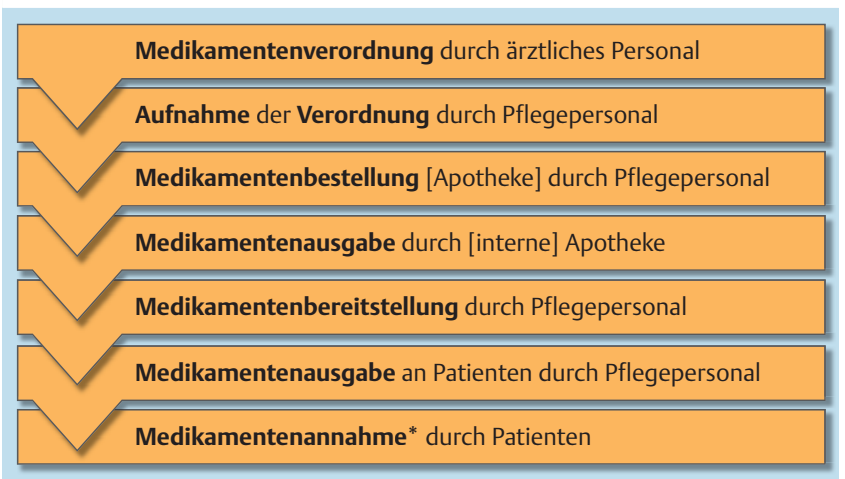

Abb. 1 Die wichtigsten in der Literatur identifizierten Schwachstellen für potenzielle Fehlschläge im Zusammenhang mit Medikamentengaben $[18,23]$.

${ }^{*}$ Cave: Die Medikamentenannahme durch den Patienten ist nicht gleichzusetzen mit der (korrekten) Medikamenteneinnahme. Hier liegt eine weitere potenzielle Schwachstelle im System.

(Beinahe-)Fehlschläge und UE aufzuspüren, führt zu einer verlässlichen Verbesserung der Patientensicherheit und der (intensivmedizinischen) Behandlungsqualität [34,54-56].

Die Schlüsselrolle der „kleinen“ Fehlschläge: Den allermeisten großen Fehlschlägen oder Katastrophen im Gesundheitswesen geht eine ganze Kette von kleinen multifaktoriellen (Beinahe-) Fehlschlägen voraus [25,34,57]. Daher ist es von essenzieller Bedeutung, diese „kleinen“ Fehlschläge und insbesondere auch die Beinahe-Fehlschläge (Warnhinweise), welche in der alltäglichen Routine auftreten, konsequent aufzuspüren, um die Wahrscheinlichkeit zukünftiger (großer) Fehlschläge vermindern zu können $[25,34]$. Auch kann die konsequente Überwachung und Analyse der Beinahe-Fehlschläge im gesamten Arbeitsumfeld zu einer verbesserten Achtsamkeit gegenüber möglichen Risiken beitragen und zu Eigeninitiativen und Systemänderungen führen, welche die Rate und das Ausmaß von Fehlschlägen reduzieren können [34]. Gerade auf einer Intensivstation treten z. B. in Hinblick auf die Medikamentengabe deutlich mehr UE als auf einer Normalstation auf, und sie sind zudem auch häufiger von erheblicher klinischer Konsequenz [58]. Dies ist v.a. durch die hohe Anzahl an Medikamentengaben zu erklären, denn diese Unterschiede relativieren sich, wenn die Anzahl der applizierten Medikamente mitberücksichtigt wird [58]. Dennoch bilden intensivmedizinisch versorgte Patienten damit eine Hochrisiko-Gruppe in Bezug auf mögliche UE und sollten daher im Fokus der Bemühungen um eine verbesserte Patientensicherheit stehen [34]. Nicht jeder (Beinahe-)Fehlschlag fügt auch tatsächlich dem Patienten einen Schaden i.S. eines UE zu [59,60]. Hieraus erwuchs die Erkenntnis, dass nicht das Aufspüren von (Beinahe-)Fehlschlägen per se die Patientensicherheit erhöht, sondern vorrangig diejenigen identifiziert werden müssen, welche einem Patienten Schaden zufügen können [59,60]. Als Beispiel seien hier im Umfeld der Beatmungsmedizin Fehlschläge z.B. in der mechanischen Kopplung zwischen Beatmungsgerät und Patient genannt, welche großen Schaden anrichten können [5].

Identifikationssysteme etablieren: Eine Möglichkeit zur Identifikation potenziell schädigender Einwirkungen auf den Patienten stellt die Verwendung sogenannter (manueller und zunehmend auch automatisierter) „Trigger“-Werkzeuge dar: bestimmte Signalwörter oder Ereignisse (z.B. die Verordnung bestimmter Medikamente oder auch pathologische Laborwerte) in der Patientenakte veranlassen eine detaillierte Analyse des Behand- lungsfalls und können so bedrohliche (Beinahe-)Fehlschläge und (potenzielle) UE zuverlässig aufspüren [33,61]. Für intensivmedizinisch versorgte Patienten existiert beispielsweise ein (manuelles) Trigger-Werkzeug (Checkliste), welches u.a. evaluiert, ob Naloxon oder Flumazenil eingesetzt wurden, ob eine Pneumonie neu diagnostiziert wurde, ob eine (Re-)Intubation erfolgte oder positive Blutkulturen verzeichnet wurden [33]. Eine pathologische Atemfrequenz ( $<10 / \mathrm{min}$ bzw. $\geq 25 / \mathrm{min}$ ) erwies sich als stärkster Prädiktor für das Auftreten eines UE bis 72 Stunden nach Entlassung von der Intensivstation [12]. Ferner wurde gezeigt, dass wiederaufgenommene Patienten auf der Intensivstation initial früher nach der Extubation von der Intensivstation entlassen wurden [17]. Als Trigger-Werkzeug bietet sich demnach an, systematisch nach pathologischen Atemfrequenzen und Unterschreiten einer bestimmten Zeitspanne nach Extubation bei allen kritisch kranken Patienten zum Zeitpunkt einer geplanten Entlassung von der Intensivstation zu suchen.

Berichtsysteme für kritische Zwischenfälle bzw. medizinische Fehler (engl. critical incident/medical error reporting system, CIRS/MERS), wie sie vom Institute of Medicine gefordert wurden, sind heute weltweit verbreitet und erfassen neben der Konsequenz auch die Wiederholungswahrscheinlichkeit eines Zwischenfalls $[1,49,56]$. Die Effektivität dieser Systeme ist jedoch erheblich eingeschränkt (so werden z. B. bestenfalls $10 \%$ aller kritischen Zwischenfälle erfasst [62]) und hängt maßgeblich von der Bereitschaft zur freiwilligen Berichterstattung und sicheren Rahmenbedingungen, aber auch vom Wissen zur korrekten Dateneingabe $a b[1,31,56,60]$. Zudem scheinen in der Intensivmedizin gerade die besonders häufig auftretenden Zwischenfälle, welche das respiratorische System bzw. die Beatmungstherapie betreffen, vom Personal unterrepräsentiert berichtet zu werden [19]. Mit „Learning From Defects“ steht ein auch auf Intensivstationen anzuwendendes Werkzeug zur Verfügung, das nicht nur (Beinahe-)Fehlschläge dokumentiert, sondern darüber hinaus die begünstigenden Faktoren identifiziert und mittels Follow-up Mechanismen gewährleistet, dass Verbesserungen in der Patientensicherheit erzielt wurden [63].

Frühwarnsysteme (engl. rapid response teams) könnten möglicherweise helfen, die Notwendigkeit intensivmedizinischer Maßnahmen bei Patienten auf Normalstation besser einzuschätzen [64]. Eine abschließende Bewertung bzgl. der Effektivität ist jedoch bisher nicht möglich: So zeigte dieses Vorgehen in einer Studie keine Verbesserungen, in einer weiteren Untersuchung wurde jedoch die Krankenhausmortalität signifikant reduziert [65]. Auch Identifikationssysteme einer inadäquaten Pharmakotherapie können die Rate an Wiederaufnahmen auf eine Intensiveinheit reduzieren [66].

\section{Förderung von Systemen zur Analyse und Diskussion von Fehlschlägen}

Morbiditäts- und Mortalitäts-Konferenzen: Diese Veranstaltungen (engl. morbidity and mortality conferences, M\&MC) wurden in weiten Teilen bereits Ende der 1980er-Jahre eingeführt und haben zum Ziel, durch Fallbesprechungen (oft in Kombination mit Autopsien) aus (zumeist) chirurgischen Fehlschlägen und UE zu lernen; hierdurch soll das (v.a. ärztliche) Personal fortgebildet und die Patientenversorgung verbessert werden [60,67]. Letzteres wurde bisher nicht wissenschaftlich belegt; gleichwohl ist das Vertrauen in die Effektivität von M\&MC hoch [60]. Nachteile der M\&MC stellen die willkürliche Auswahl (und Nicht-Auswahl) der Fälle, die begrenzte Anzahl an Teilnehmern, die Gefahr eines Rückschaufehlers sowie die geringe UE-Fallzahl dar - damit ran- 
gieren sie biometrisch bestenfalls unter der niedrigen Evidenzkategorie „Fallserien“ [60].

Direkte Beobachtung der Patientenversorgung: Unter direkter arbeitsplatzbasierter Beobachtung und (Video-)Aufzeichnung der Patientenversorgung (nach entsprechender Aufklärung und Einverständniserklärung aller Beteiligten) lassen sich in Echtzeit (Beinahe-)Fehlschläge analysieren [60]. Dies bietet v.a. auch für das komplexe Umfeld einer Intensivstation eine interessante Möglichkeit, aus den Beobachtungen und Analysen direkte Konsequenzen zur Verbesserung der Patientensicherheit zu ziehen [13]. Beobachter erhöhen hier nicht nur die berichtete Anzahl der (Beinahe-)Fehlschläge gegenüber den Personaleinschätzungen, sondern decken v.a. die vom Personal offenbar unterschätzten besonders häufigen Ereignisse, welche das respiratorische System bzw. die Beatmungstherapie betreffen, zuverlässig auf [19]. Diese Methode ist jedoch auf Analysen an der „Speerspitze“ beschränkt, unterliegt dem Hawthorne-Effekt, ist sehr zeit- und ressourcenaufwändig und auf sichere Rahmenbedingungen angewiesen, da die Aufzeichnungen potenziell gegen das Personal verwendet werden könnten [60].

Analyse von Behandlungsfehlerklagen: Ein interessantes Werkzeug stellt die Analyse von Klageverfahren bzgl. medizinischen Behandlungsfehlern (engl. malpractice claims) dar: So existiert eine große Anzahl an Patientenakten, Zeugen- und Sachverständigenaussagen und damit die Möglichkeit, auch Beinahe-Fehlschläge zu analysieren $[60,68]$. In Deutschland böte sich zudem an, die an den medizinischen Dienst der Krankenversicherung (MDK) gemeldeten Verdachtsfälle von Behandlungsfehlern zu analysieren und gleichzeitig eine offenere Kultur des Umgangs mit Fehlschlägen voranzutreiben: In den Jahren 2011 bzw. 2012 wurden rund 12500 Verdachtsfälle gemeldet, wovon in rund einem Drittel der ärztlichen Fälle und etwa jedem zweiten pflegerischen Verdachtsfall ein tatsächlicher Behandlungsfehler durch den MDK gutachterlich bestätigt wurde $[69,70]$. Nachteile dieser Verfahren sind die Selektivität, die fehlende Standardisierung und neuerlich die Gefahr eines Rückschaufehlers [60]. Zudem fehlen hier zumeist die sicheren Rahmenbedingungen gänzlich, da für die Beteiligten teils erhebliche negative juristischen Konsequenzen drohen.

Die besondere Dringlichkeit für die Pneumologie aus (Beinahe-) Fehlschlägen zu lernen, lässt sich in diesem Zusammenhang u.a. daran ablesen, dass sie anhand valider Daten aus den USA (Beobachtungszeitraum 1991 -2005) zusammen mit der Gastroenterologie unter allen nicht-operativen Disziplinen (inklusive der Notfallmedizin) der höchsten Rate an Verdachtsfällen von Behandlungsfehlern ausgesetzt ist [71].

Umfassendes Qualitätsmanagement: Dieses Verfahren (engl. total quality management) zielt zur Reduktion von UE darauf ab, die ärztliche Verordnungspraxis im Hinblick auf Fehlerquellen (z.B. aufgrund Unleserlichkeit) zu optimieren [18]. Hier konnte gezeigt werden, dass Systeme, welche heute als computergestützte ärztliche Verordnung (engl. computerized physician order entry) als Teil der so genannten elektronischen Gesundheitsakte bekannt sind, erhebliche Verbesserungen schaffen [72,73]. Eine wegweisende Arbeit aus dem Jahre 1991 wurde 2005 neuerlich publiziert, und in dem zugehörigen Kommentar konstatiert Battles, dass die Weiterentwicklung dieser Systeme (z. B. automatisierte Trigger-Werkzeuge) in den letzten Jahren die erhofften Fortschritte in der Patientensicherheit erzielen konnte - die vollständige Implementierung der optimierten Systeme in die tägliche Routine jedoch der zwingende nächste Schritt sein muss [55]. Wesentliche Anforderungen an elektronischen Ge- sundheitsakten sind zum einen, dass sie umgehenden Zugriff auf alle Patienten relevanten Daten ermöglichen, und zum anderen, dass sicherheitsrelevante Patientendaten gespeichert und analysiert werden können [34]. Da zunehmend gesundheitsrelevante Daten zwischen verschiedenen Stellen im Gesundheitswesen ausgetauscht werden müssen, sind Standards für den Datenaustausch (z. B. das DICOM-Format in der medizinischen Bildgebung - engl. digital imaging and communications in medicine) unerlässlich [34]. Ein weiterer wertvoller Aspekt des umfassenden Qualitätsmanagements kann die Durchführung so genannter (ärztlicher) „Peer Review“ Verfahren mit Vorort-Besichtigungen sein [74].

Keinesfalls dürfen die potenziellen Chancen dieses Verfahrens in dem Sinne missverstanden werden, als das heute im Gesundheitswesen allzu oft der (ökonomische) Begriff „Qualitätsmanagement“ zur Förderung einer bürokratisch ausgerichteten Institution mit immer härteren ökonomischen Zielvorgaben sowie Gewinnmaximierung missbraucht wird und so häufig falsche Prioritäten setzt [37].

Interdisziplinäre/Multiprofessionelle Zusammenarbeit: Durch die Komplexität des Behandlungsumfeldes, etlicher beteiligter Disziplinen und Berufsgruppen, aber auch durch ständig wechselnde Teammitglieder stellt die Zusammenarbeit, Kommunikation und Rollenfindung auf der Intensivstation höchste Anforderungen an alle Beteiligten und birgt erhebliche Spannungsfelder [75, 76].

Das konzeptuelle Bezugsystem des modifizierten Eindhoven-Modells verdeutlicht beispielhaft, wie komplex das Gesundheitswesen in Bezug auf (Beinahe-)Fehlschläge betrachtet werden muss: Unausweichliche menschliche/personelle, technische und organisatorische (Beinahe-)Fehlschläge erzeugen ein Szenario mit hohem Gefährdungspotential, welches nur durch geeignete Schutzmechanismen nicht zwangsläufig in einem UE resultiert [77]. Eine funktionierende interdisziplinäre und multiprofessionelle Zusammenarbeit aller Beteiligten ist essenziell, um aus (Beinahe-)Fehlschlägen lernen zu können und die Patientensicherheit zu verbessern [77]. Möglichkeiten hierzu ergeben sich nach diesem Modell an drei Stellen: 1 . bei Sicherheitsmechanismen, welche auf sorgfältig erstellten personellen (z. B. Führungsstil), technischen (z.B. elektronische Gesundheitsakte) und organisatorischen (z.B. Behandlungspfade) Verfahrensabläufen beruhen, 2. bei Schutzmechanismen des Gesamtsystems, welche eng in die Patientenversorgung eingewoben sind und aufkommende Abweichungen zuverlässig erkennen (z.B. computergestützte ärztliche Verordnungssysteme), und 3. Schutzmechanismen, welche durch direkt am Patienten tätiges Personal (neben ärztlichem Personal häufig Pflegefachkräfte, aber zunehmend auch Atmungstherapeuten) erbracht werden und so direkt an der „Speerspitze“ (Beinahe-)Fehlschläge identifizieren und abmildern/verhindern können [77]. Gerade die wertvolle und tragende Rolle des nicht-ärztlichen Personals (i.e. Pflegefachkräfte und Atmungstherapeuten) in diesem Lernprozess zur Verbesserung der Patientensicherheit sollte hierbei nicht vernachlässigt werden [78].

Um Missverständnissen vorzubeugen und um eine allseits verständliche Diskussion und Analyse von möglichen Fehlschlägen unter allen Berufsgruppen im Gesundheitswesen betreiben zu können, kommt einer gemeinsamen und möglichst eindeutigen Terminologie (auch in Bezug auf die internationale Klassifikation der Krankheiten und Gesundheitsprobleme durch die Weltgesundheitsorganisation) eine herausragende Bedeutung zu [34]. Zudem erarbeitete die Weltgesundheitsorganisation seit 2005 


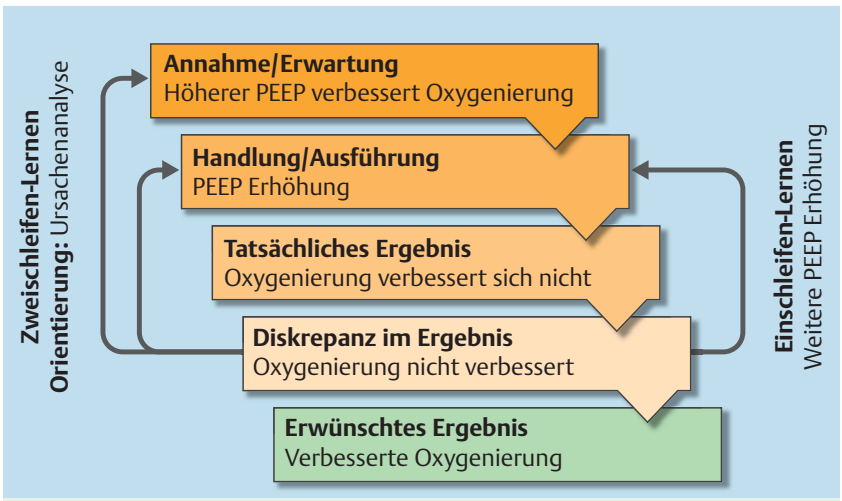

Abb.2 Vergleich zwischen Ein- bzw. Zweischleifen-Lernen am Beispiel einer schwierigen Beatmungssituation in Anlehnung an Reason et al. [37]. Auf dem Boden der (als allgemeingültig verstanden) Annahme, dass eine Erhöhung des positiven end-exspiratorischen Drucks (PEEP) zu einer Verbesserung der Oxygenierung führt, wird der PEEP bei einem intubierten Patienten mit zunehmender Oxygenierungsstörung mit dem erwarteten Ergebnis einer verbesserten Oxygenierung erhöht. Diese bleibt allerdings aus. Beim Einschleifen-Lernen wird nun unreflektiert der PEEP weiter erhöht. Beim Zweischleifen-Lernen erfolgt i. S. einer Orientierungsphase eine Ursachenanalyse: Nach Auskultation und Röntgen des Thorax zeigt sich ein unerkannter Spannungspneumothorax als Grund der diskrepanten Ergebnisse und durch Modifikation der Handlung (i. e. Thoraxdrainagenanalage) kann das erwünschte Ergebnis einer verbesserten Oxygenierung (nebst der vernachlässigten Ventilation) zielführend erreicht werden.

eine internationale Klassifikation für Patientensicherheit (engl. international classification for patient safety) [79]. Die medizinische Nationalbibliothek in den USA leistet hierzu - v.a. durch die von ihr geführte frei zugängliche Meta-Datenbank PubMed einen national wie global entscheidenden Beitrag, welcher laufend weiter ausgebaut wird [34]. Auch muss ein breiter und barrierefreier Zugang (z.B. keine Einschränkung durch kostenpflichtige Zugriffsrechte auf Publikationen) für alle potenziell Interessierten und Betroffenen zu bereits bekannten Gefahrenquellen oder Lösungsansätzen realisiert werden [34].

Systematische Datenanalyse: In Feldern mit hohem Gefährdungspotenzial - z.B. im Gesundheitswesen - provozieren vergleichbare Situationen bei völlig unterschiedlichen Menschen stets aufs Neue die gleiche Kategorie von Fehlschlägen $[37,80]$. Daher kommt der systematischen Datenanalyse inklusive der Anwendung eines standardisierten Vorgehens anhand eines Protokolls entscheidende Bedeutung zu [43]. So konnte für UE im Zusammenhang mit Medikamentengabe gezeigt werden, dass eine systematische Kontrolle und Analyse (z. B. der Verordnungen anhand der Patientenakte) einem freiwilligen Berichterstatten möglicher UE überlegen ist [18,34,81]. Bei Zugriff auf Patientenakten muss jedoch beachtet werden, dass eine lückenhafte Dokumentation zu erheblichen Verzerrungen führen kann; hier kann die elektronische Gesundheitsakte zu Verbesserungen beitragen [60].

Das modifizierte Gesundheitswesen-Modell nach Haddon besagt, dass ein UE zu drei Zeitpunkten in der Wahrscheinlichkeit seines Auftretens und in seinem Ausmaß beeinflusst werden kann: vor - während - nach des UE $[80,82]$. Diese Stellen müssen jeweils für die Aspekte Betroffener/Ausführung/Umfeld separat analysiert werden $[80,82]$. Das Modell lässt sich am Beispiel einer im Schockraum (=Umfeld) aufgrund eines seitenverkehrt projizierten Thorax-Röntgenbildes auf der falschen Seite angelegten Thoraxdrainage (=Ausführung) bei einer Patientin mit HämatoPneumothorax (= Betroffene) gut illustrieren [80].
Auch die Rückmeldungen von Patienten und deren Angehörigen müssen einbezogen und auf deren Anliegen eingegangen werden [34]. Teils weichen die Gewichtungen der Patienten und des Personals erheblich voneinander ab: So beschweren sich Patienten an erster Stelle über den klinischen Ablaufprozess (Prozeduren, Diagnosestellung), das Verhalten des Personals (unangemessen, unhöflich) sowie die Ressourcen der Einrichtung (Bettenkapazität, Service), während das Personal an kritischen Ereignissen zuvorderst Patientenunfälle, Medikamente und Medizinprodukte benennt [56].

Eine Möglichkeit den Teufelskreis des „Syndroms des vulnerablen Systems" zu durchbrechen, besteht für medizinische Einrichtungen darin, das weit verbreitete [57] Einschleifen-Lernen (engl. single loop learning) durch das Zweischleifen-Lernen (engl. double loop learning) zu ersetzen: Bei letzterem wird systematisch versucht, die Diskrepanz zwischen tatsächlichem und erwünschtem Ergebnis durch eine (interprofessionelle) Orientierungsphase und Ursachenanalyse zu verstehen und nicht ausschließlich die unreflektierte Handlung (welche unweigerlich erneut zu einem Fehlschlag führen wird) abermals auszuführen [25,37]. @ Abb. 2 erläutert die beiden Modelle an einem klinischen Beispiel. Das Einschleifen-Lernen zeichnet sich so u.a. durch provisorische Lösungsansätze mit dem Ziel der schnellen (anstelle der nachhaltigen und systematischen) Problemlösung aus [25,57]. Das im Krankenhaus äußerst selten angewendete Zweischleifen-Lernen (z.B. $<10 \%$ der pflegerischen Problemlösungsansätze) bietet nicht nur eine dauerhafte Problemlösung, sondern auch die Vorteile einer höheren Produktivität und höheren Zufriedenheit seitens der Patienten (durch verbesserte Behandlungsbedingungen) und des Personals (durch das Erfolgserlebnis) [25]. Die Hauptgründe für die seltene Anwendung im Gesundheitswesen sind: die Doktrin der hohen individuellen personellen Verantwortlichkeit (und die hierdurch gepflegte Kultur, keine Hilfe bei Anderen zu suchen), die hohe Arbeitslast und Prozessverdichtung sowie die fehlende Rückkopplung zwischen patientennahen (z. B. Pflegefachkraft) und -fernen (z. B. leitende Verwaltungsangestellte) Arbeitsbereichen innerhalb der Einrichtung [25].

Als wesentlicher Schritt zur Verbesserung der Patientensicherheit gilt heute der Aufbau einer landesweiten Informationstechnologie-Infrastruktur, deren Basis die elektronische Gesundheitsakte, weitere medizinische Anwendungsprogramme, standardisierte Vorgehensweisen und gemeinsame Leitlinien bilden und die in Form einer Zusammenarbeit privater Unternehmen und Regierungsbehörden mit klar definierten politischen und rechtlichen Rahmenbedingungen realisiert werden sollte [34]. Weiterentwicklungen der elektronischen Gesundheitsakte verbessern die Patientensicherheit u.a. dadurch, dass mehr und mehr Systeme und Möglichkeiten zur systematischen (automatisierten) Datenanalyse nicht nur von UE, sondern auch von Beinahe-Fehlschlägen etabliert werden [34].

Schließlich lassen sich auch mittels der klinischen Überwachung (engl. clinical surveillance) wertvolle Daten gewinnen: Die prospektive, aktive Erhebung (z.B. postoperativer Komplikationen) ermöglicht die Beurteilung bzgl. der Effektivität spezifischer Interventionen und kann zur Reduktion von definierten UE eingesetzt werden [60]. Diese Daten werden teilweise auch im Rahmen von (randomisierten, kontrollierten) Studien generiert; hierbei stellt ein vorzeitiger Studienabbruch, welcher nicht auf gehäuften UE oder einer nachgewiesenen Überlegenheit beruht, ein relevantes Problem dar, da wichtige Informationen bzgl. möglicher UE verloren gehen [83]. 


\section{Etablierung von geschützten Experimenten}

Um aus (Beinahe-)Fehlschlägen lernen zu können, muss offengelegt werden, was nicht funktioniert; hierzu müssen zur richtigen Zeit und am richtigen Ort geschützte Experimente (i. e. „Pilotprojekte“) initiiert werden [41]. Unter Wissenschaftlern ist unstrittig, dass eine große Zahl an Forschungsprojekten nicht den erhofften Erfolg bringt, sondern nicht wenige davon scheitern; dennoch bleiben sie hochmotiviert, weil sie Scheitern als elementaren Bestandteil im Streben nach Verbesserung akzeptieren und zumeist wichtige Erkenntnisse daraus gewinnen [41,84]. Um Grenzen auszuloten und Erkenntnisgewinn erzielen zu können, sollten (kleine) Pilotprojekte folgende Kriterien berücksichtigen: Ablauf unter realen (nicht optimierten) Bedingungen (Personal, Ausrüstung, Umgebung etc.), erkenntnisgewinn- und nicht erfolgsorientiert, Einbindung aller Interessenvertreter und schließlich die entscheidende Frage, ob durch das Pilotprojekt positive und dauerhafte Änderungen erzielt worden sind [41]. Nur allzu leicht wird übersehen, dass Lernen aus Erfolgen ungleich schwieriger (und demnach noch unwahrscheinlicher) ist als aus (Beinahe-)Fehlschlägen, wo die elementare Frage des „Warum?“ quasi auf der Hand liegt - ein Erfolg wird im Gegensatz dazu meist als gegeben hingenommen, verleitet zu verminderter (Selbst-)Reflexion und zur (Selbst-)Überschätzung [84]. Als Leitspruch einer Institution, welche für sich verinnerlicht hat, aus Fehlschlägen zu lernen, könnte demnach die positive Auslegung des folgenden Zitats von Samuel Beckett aus der Novelle Worstward Ho gelten: "All of old. Nothing else ever. Ever tried. Ever failed. No matter. Try again. Fail again. Fail better." [85].

\section{Danksagung \\ $\nabla$}

Mein aufrichtiger Dank gilt Prof. Dr. Amy C. Edmondson (Harvard Business School, Harvard University, Boston, USA) für ihre Inspiration und die wertvolle Unterstützung zu diesem Manuskript vor Drucklegung.

\section{Interessenkonflikt}

Der Autor gibt an, dass kein Interessenkonflikt besteht.

\section{Literatur}

1 Kohn LT, Corrigan JM, Donaldson MS, eds. To Err Is Human: Building a Safer Health System. The National Academies Press; 2000: Im Internet: http://www.nap.edu/openbook.php?record_id=9728 Stand: 03.06.2013

2 Vincent $C$. Understanding and responding to adverse events. N Engl J Med 2003; 348: 1051 - 1056

3 Zhan C, Miller MR. Excess length of stay, charges, and mortality attributable to medical injuries during hospitalization. J Am Med Assoc 2003; 290: $1868-1874$

4 Vlayen A, Verelst S, Bekkering GE et al. Incidence and preventability of adverse events requiring intensive care admission: a systematic review. J Eval Clin Pract 2012; 18: 485 - 497

5 Stieglitz S, George S, Priegnitz C et al. Life-threatening Events in Respiratory Medicine: Misconnections of Invasive and Non-invasive Ventilators and Interfaces. Pneumologie 2013; 67: 228-232

6 Frey B, Kehrer B, Losa M et al. Comprehensive critical incident monitoring in a neonatal-pediatric intensive care unit: experience with the system approach. Intensive Care Med 2000; 26: 69-74

7 Bracco D, Favre JB, Bissonnette $B$ et al. Human errors in a multidisciplinary intensive care unit: a 1-year prospective study. Intensive Care Med 2001; 27: 137-145
8 Thomas EJ, Studdert DM, Burstin HR et al. Incidence and types of adverse events and negligent care in Utah and Colorado. Med Care 2000; 38: $261-271$

9 Vincent C, Neale G, Woloshynowych M. Adverse events in British hospitals: preliminary retrospective record review. BMJ 2001; 322: 517 519

10 Wilson RM, Runciman WB, Gibberd RW et al. The Quality in Australian Health Care Study. Med J Aust 1995; 163: 458-471

11 Brennan TA, Leape $L L$, Laird NM et al. Incidence of adverse events and negligence in hospitalized patients. Results of the Harvard Medical Practice Study I. N Engl J Med 1991; 324: 370 - 376

12 Chaboyer W, Thalib L, Foster $M$ et al. Predictors of adverse events in patients after discharge from the intensive care unit. Am J Crit Care 2008; 17: $255-263$

13 Donchin Y, Gopher D, Olin $M$ et al. A look into the nature and causes of human errors in the intensive care unit. Crit Care Med 1995; 23: $294-$ 300

14 Abramson NS, Wald KS, Grenvik AN et al. Adverse occurrences in intensive care units. J Am Med Assoc 1980; 244: 1582 - 1584

15 Wright D, Mackenzie SJ, Buchan I et al. Critical incidents in the intensive therapy unit. Lancet 1991; 338: 676-678

16 Rosenberg $A L$, Watts $C$. Patients readmitted to ICUs* : a systematic review of risk factors and outcomes. Chest 2000; 118: $492-502$

17 Metnitz PGH, Fieux F, Jordan B et al. Critically ill patients readmitted to intensive care units-lessons to learn? Intensive Care Med 2003; 29: $241-248$

18 Bates DW, Leape LL, Petrycki S. Incidence and preventability of adverse drug events in hospitalized adults. J Gen Intern Med 1993; 8: 289-294

19 Capuzzo M, Nawfal I, Campi M et al. Reporting of unintended events in an intensive care unit: comparison between staff and observer. BMC Emerg Med 2005; 5: 3

20 Leape LL, Brennan TA, Laird N et al. The nature of adverse events in hospitalized patients. Results of the Harvard Medical Practice Study II. N Engl J Med 1991; 324: 377 - 384

21 Kopp BJ, Erstad BL, Allen ME et al. Medication errors and adverse drug events in an intensive care unit: direct observation approach for detection. Crit Care Med 2006; 34: 415-425

22 Welters ID, Gibson J, Mogk M et al. Major sources of critical incidents in intensive care. Crit Care 2011; 15: R232

23 Edmondson AC. Learning from Mistakes is Easier Said Than Done: Group and Organizational Influences on the Detection and Correction of Human Error. J Appl Behav Sci 1996; 32: 5-28

24 Reinertsen JL. Let's talk about error. BMJ 2000; 320: 730

25 Edmondson AC. Learning from failure in health care: frequent opportunities, pervasive barriers. Qual Saf Health Care 2004; 13 (Suppl. 2): ii $3-9$

26 Stoller JK. Implementing change in respiratory care. Respir Care 2010; 55: $749-757$

27 Anders ME, Evans DP. Comparison of PubMed and Google Scholar literature searches. Respir Care 2010; 55: 578-583

28 Nourbakhsh E, Nugent $R$, Wang $H$ et al. Medical literature searches: a comparison of PubMed and Google Scholar. Heal Inf Libr J 2012; 29: $214-222$

29 Le Dévic $N$. Council of Europe recommendation and glossary on patient and medication safety: CPME Info 86-2006. Im Internet: http://cpme.dyndns.org:591/database/2006/Info.2006-086.enonly.pdf Stand: 03.06.2013

30 Sheikh A, Hurwitz B. Setting up a database of medical error in general practice: conceptual and methodological considerations. Br J Gen Pract $2001 ; 51: 57-60$

31 Vincent C, Stanhope N, Crowley-Murphy M. Reasons for not reporting adverse incidents: an empirical study. J Eval Clin Pract 1999; 5: $13-21$

32 Edmondson AC, Schein EH. Teaming: How Organizations Learn, Innovate, and Compete in the Knowledge Economy. 1. Aufl. San Francisco: John Wiley \& Sons; 2012

33 Resar RK, Rozich JD, Classen D. Methodology and rationale for the measurement of harm with trigger tools. Qual Saf Health Care 2003; 12 (Suppl. 2): ii39-45

34 Aspden P, Corrigan JM, Wolcott J, Erickson SM, eds. Patient Safety: Achieving a New Standard for Care. The National Academies Press; 2004: Im Internet: http://www.nap.edu/openbook.php?record_id=10863 Stand: 03.06.2013

35 Boyle D, O'Connell D, Platt FW et al. Disclosing errors and adverse events in the intensive care unit. Crit Care Med 2006; 34: 1532 - 1537 
36 Cannon MD, Edmondson AC. Failing to Learn and Learning to Fail (Intelligently). Long Range Plann 2005; 38: 299-319

37 Reason JT, Carthey J, de Leval MR. Diagnosing "vulnerable system syndrome": an essential prerequisite to effective risk management. Qual Heal Care 2001; 10 (Suppl. 2): ii21 - 25

38 Nottingham J. Medical errors. Perhaps blame-free culture is needed in NHS to reduce errors. BMJ 2001; 322: 1422

39 Turton C. Medical errors. Media tend to link error with blame. BMJ $2001 ; 322: 1422$

40 Reason J. Human error: models and management. BMJ 2000; 320: $768-770$

41 Edmondson AC. Strategies of learning from failure. Harv Bus Rev 2011; 89: $48-55,137$

42 Perrow C. Normal Accidents: Living with High-Risk Technologies. Updated. 1. Aufl. Chichester: Princeton University Press; 1999

43 Vincent C, Taylor-Adams S, Chapman EJ et al. How to investigate and analyse clinical incidents: clinical risk unit and association of litigation and risk management protocol. BMJ 2000; 320: 777-781

44 Garvin DA, Edmondson AC, Gino F. Is yours a learning organization? Harv Bus Rev 2008; 86: 109-116, 134

45 Milligan F, Bird D. Adverse health-care events: Part 4. Challenge of a blame-free culture. Prof Nurse 2003; 18: 705 - 709

46 Tucker AL, Nembhard IM, Edmondson AC. Implementing New Practices: An Empirical Study of Organizational Learning in Hospital Intensive Care Units. Manag Sci 2007; 53: 894-907

47 Wise JA, Hopkin VD, Stager P, eds. Verification and Validation of Complex Systems: Human Factors Issues. 1. Aufl. New York, Heidelberg: Springer; 2010: 402

48 Edmondson AC. The competitive imperative of learning. Harv Bus Rev 2008; 86: 60-67, 160

49 Bird D, Milligan F. Adverse health-care events: Part 2. Incident reporting systems. Prof Nurse 2003; 18: $572-575$

50 Bird D, Milligan F. Adverse health-care events: Part 3. Learning the lessons. Prof Nurse 2003; 18: $621-625$

51 Ramani S, Krackov SK. Twelve tips for giving feedback effectively in the clinical environment. Med Teach 2012; 34: 787-791

52 Edmondson AC. Framing for Learning: Lessons in Successful Technology Implementation. Calif Manage Rev 2003; 45: 34-54

53 Shortell SM, Zimmerman JE, Rousseau DM et al. The performance of intensive care units: does good management make a difference? Med Care 1994; 32: 508-525

54 Curtis JR, Cook DJ, Wall RJ et al. Intensive care unit quality improvement: a "how-to" guide for the interdisciplinary team. Crit Care Med 2006; 34: $211-218$

55 Classen D, Pestotnik S, Evans $R$ et al. Computerized surveillance of adverse drug events in hospital patients*. Qual Saf Health Care 2005; 14: $221-226$

56 De Feijter JM, de Grave WS, Muijtjens AM et al. A comprehensive overview of medical error in hospitals using incident-reporting systems, patient complaints and chart review of inpatient deaths. Plos One 2012; 7: e31125

57 Tucker AL, Edmondson AC. Why Hospitals Don't Learn from Failures: Organizational and Psychological Dynamics that Inhibit System Change. Calif Manage Rev 2003; 45: 55-72

58 Cullen DJ, Sweitzer BJ, Bates DW et al. Preventable adverse drug events in hospitalized patients: a comparative study of intensive care and general care units. Crit Care Med 1997; 25: 1289-1297

59 Rozich JD, Haraden CR, Resar RK. Adverse drug event trigger tool: a practical methodology for measuring medication related harm. Qual Saf Health Care 2003; 12: 194-200

60 Thomas EJ, Petersen LA. Measuring errors and adverse events in health care. J Gen Intern Med 2003; 18: 61 -67

61 Classen DC, Lloyd RC, Provost $L$ et al. Development and Evaluation of the Institute for Healthcare Improvement Global Trigger Tool. J Patient Saf 2008; 4: 169-177
62 Sari AB-A, Sheldon TA, Cracknell A et al. Sensitivity of routine system for reporting patient safety incidents in an NHS hospital: retrospective patient case note review. BMJ 2007; 334: 79

63 Pronovost PJ, Holzmueller CG, Martinez E et al. A practical tool to learn from defects in patient care. Jt Comm J Qual Patient Saf 2006; 32: 102 108

64 McGloin H, Adam SK, Singer M. Unexpected deaths and referrals to intensive care of patients on general wards. Are some cases potentially avoidable? J R Coll Physicians Lond 1999; 33: 255-259

65 McGaughey J, Alderdice F, Fowler R et al. Outreach and Early Warning Systems (EWS) for the prevention of intensive care admission and death of critically ill adult patients on general hospital wards. Cochrane Database Syst Rev 2007; 3: CD005529

66 Stewart S, Voss DW. A study of unplanned readmissions to a coronary care unit. Heart Lung J Crit Care 1997; 26: 196-203

67 Campbell WB. Surgical morbidity and mortality meetings. Ann R Coll Surg Engl 1988; 70: $363-365$

68 Cheney FW, Posner K, Caplan RA et al. Standard of care and anesthesia liability. J Am Med Assoc 1989; 261: 1599-1603

69 MDS - Medizinischer Dienst des Spitzenverbandes Bund der Krankenkassen e.V. Jahresstatistik 2011 zur Behandlungsfehler-Begutachtung der MDK-Gemeinschaft. Im Internet: http://www.mdk.de/media/pdf/ Bericht_2011_Behandlungsfehler-Begutachtung_MDK-Gemeinschaft. pdf Stand: 03.06.2013

70 MDS - Medizinischer Dienst des Spitzenverbandes Bund der Krankenkassen e.V. Jahresstatistik 2012 zur Behandlungsfehler-Begutachtung der MDK-Gemeinschaft. Im Internet: http://www.mdk.de/media/pdf/ 8_-_Bericht_BHF-Begutachtung_2012_final.pdf. Stand: 03.06.2013

71 Jena $A B$, Seabury $S$, Lakdawalla $D$ et al. Malpractice risk according to physician specialty. N Engl J Med 2011; 365: 629-636

72 Classen DC, Pestotnik SL, Evans RS et al. Computerized surveillance of adverse drug events in hospital patients. J Am Med Assoc 1991; 266: 2847-2851

73 Bates DW. Using information technology to reduce rates of medication errors in hospitals. BMJ 2000; 320: 788-791

74 Bundesärztekammer. Curriculum Ärztliches Peer Review. Im Internet: http://www.bundesaerztekammer.de/page.asp?his=1.120.1116.9069 Stand: 03.06.2013

75 Hawryluck LA, Espin SL, Garwood KC et al. Pulling together and pushing apart: tides of tension in the ICU team. Acad Med 2002; 77: 73-76

76 Lingard L, Espin S, Evans C et al. The rules of the game: interprofessional collaboration on the intensive care unit team. Crit Care 2004; 8 : R403-408

77 Henneman EA, Gawlinski A. A "near-miss" model for describing the nurse's role in the recovery of medical errors. J Prof Nurs 2004; 20: $196-201$

78 Milligan F, Bird D. Adverse health-care events: Part 1. The nature of the problem. Prof Nurse 2003; 18: 502-505

79 Sherman H, Castro G, Fletcher $M$ et al. Towards an International Classification for Patient Safety: the conceptual framework. Int J Qual Heal Care 2009; 21: 2-8

80 Brasel KJ, Layde PM, Hargarten S. Evaluation of error in medicine: application of a public health model. Acad Emerg Med 2000; 7: 12981302

81 Bennett BS, Lipman AG. Comparative study of prospective surveillance and voluntary reporting in determining the incidence of adverse drug reactions. Am J Hosp Pharm 1977; 34: 931 - 936

82 Haddon WJr. A logical framework for categorizing highway safety phenomena and activity. J Trauma 1972; 12: $193-207$

83 Kasenda B, von Elm EB, You J et al. Learning from failure-rationale and design for a study about discontinuation of randomized trials (DISCO study). BMC Med Res Methodol 2012; 12: 131

84 Gino F, Pisano GP. Why leaders don't learn from success. Harv Bus Rev 2011; 89: 68 -74, 137

85 Beckett S. Worstward Ho. 4. Aufl. London: John Calder; 1999 